\title{
IMPACT OF BAQ LEVEL ON INSAR PERFORMANCE OF RADARSAT-2 EXTENDED SWATH BEAM MODES
}

\author{
Jayson Eppler ${ }^{(1)}$, Mike Kubanski ${ }^{(1)}$ \\ ${ }^{(1)}$ MDA Systems Ltd., 13800 Commerce Parkway, Richmond, British Columbia, Canada, V6V 2J3, \\ Email:jeppler@mdacorporation.com
}

\begin{abstract}
RADARSAT-2 Wide Ultra-Fine, Wide Multi-Look Fine and Extra-Fine modes are extended swath modes which employ 2-bit Block Adaptive Quantization (BAQ) compression for data downlink rather than 3-bit BAQ which is used for most other RADARSAT-2 modes. This results in a higher level of quantization noise for these extended swath modes. We have developed a method for simulating 2-bit BAQ derived products by decoding 3-bit BAQ encoded data with alternate decode tables. This method was applied to several multitemporal 3-bit BAQ InSAR stacks to generate corresponding simulated 2-bit datasets. These matching datasets were processed in parallel using Homogenous Distributed Scatterer (HDS)-InSAR to derive multitemporal surface deformation estimates. A comparison of both intermediate and final deformation estimates show substantially similar results with the exception of low backscatter distributed targets such as asphalted surfaces in urban settings.
\end{abstract}

\section{INTRODUCTION}

RADARSAT-2 is a commercial synthetic aperture radar (SAR) satellite capable of acquiring data in a variety of modes that include single beam (stripmap), ScanSAR and spotlight beam configurations along with co-, cross, dual- or quad-polarization configurations [1]. This variety of modes results in significant variability in downlink data-rate requirements and therefore the satellite employs a flexible BAQ implementation with different BAQ bit levels assigned to the various modes.

Several modes are available that provide single look complex (SLC) products which are generated using 2bit BAQ compressed raw downlinked data. These extended swath modes have the advantage of providing significantly wider swath coverage at the same nominal resolution as their 3-bit counterparts at the expense of higher noise levels due to the increased compression level of the raw data. The 2-bit BAQ wide modes include Wide Ultra-Fine, Wide Multi-Look Fine and the recently added Extra-Fine mode with nominal swath extents of 50, 90 and $125 \mathrm{~km}$ respectively.

The extended swath modes are attractive for many applications due to the combination of resolution and spatial coverage. However the impact of the higher quantization noise associated with a reduced BAQ bit level should be considered when selecting a mode for a given application. InSAR based applications have the potential to be particularly sensitive to quantization noise due to their reliance on the complex SLC phase [2]. For this reason we have derived a method to directly study the effect of BAQ bit level on InSAR derived surface estimates.

RADARSAT-2 raw pulse returns are BAQ encoded to the prescribed bit level on the spacecraft and downlinked for subsequent processing. These BAQ encoded data segments are decoded during processing using decode tables which are designed to achieve near unity gain across all signal levels. MDA has developed a simulation method which utilizes alternate decoding tables to decode 3-bit BAQ encoded downlinked pulse returns to closely approximate 2-bit unity gain BAQ encoded/decoded pulse returns. This allows for generation of both 3-bit BAQ derived SLCs and corresponding simulated 2-bit BAQ derived SLCs from the same downlinked 3-bit BAQ data as shown in Fig.1. The simulation therefore represents a test-bed for studying the impact of 2-bit BAQ on a variety of SAR applications using already acquired 3-bit BAQ scenes.

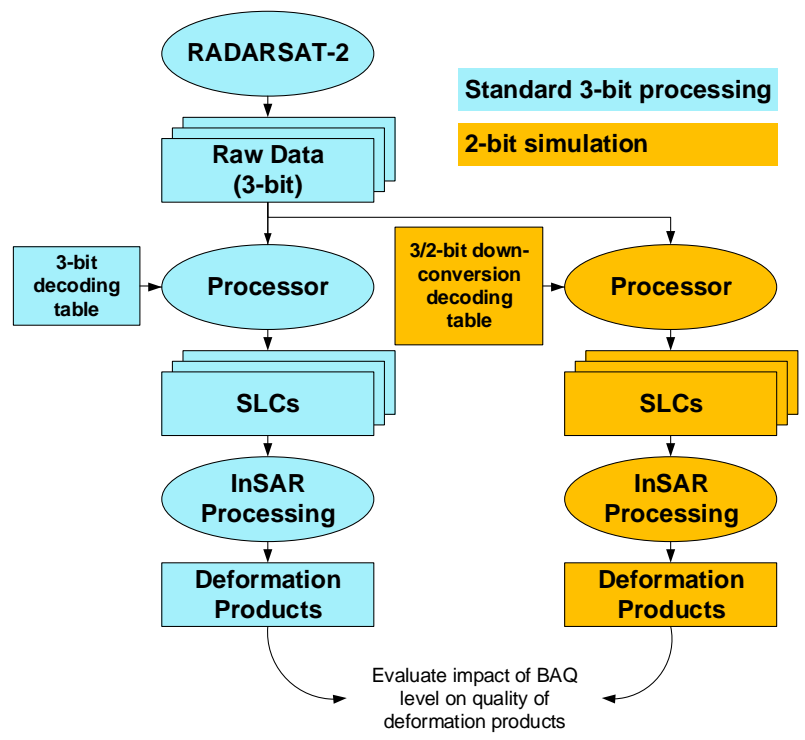

Figure 1, 2-bit BAQ simulation concept overview.

In this work we use the test-bed generated data to study the impact of BAQ bit-level on the performance of multi-temporal InSAR surface displacement monitoring. 
Multi-temporal stacks of RADARSAT-2 SLC data are processed both with 3-bit and simulated 2-bit decoding tables and then processed to derive multi-temporal surface displacement estimates. InSAR results are generated using HDS-InSAR which exploits both persistent point and coherent distributed scatterers by using adaptive multi-looking of statistically homogenous pixel neighbourhood.

\section{RADARSAT-2 BAQ ENCODING/DECODING}

The goal of raw data quantization is to achieve a prescribed bit per sample encoding for data downlink while providing uniform gain and good digitization noise ratio (DNR) over a broad range of signal levels. Raw SAR pulse return signals are expected to be locally Gaussian in distribution with variance that continuously varies both in range and azimuth. BAQ uses a set of quantization tables with each table optimized for gain and DNR performance over a narrow range of signal levels. Data is quantized in range blocks of $\mathrm{I}$ and $\mathrm{Q}$ values where the variance of each block is used to estimate the local signal level and assign the best matched table for encoding. The selected table identifier is stored with the encoded data block so that the correct corresponding decode table can be applied to the block during decoding.

Following BAQ encoding, each raw sample has a pair of assigned values corresponding to the assigned table identifier and the assigned BAQ encoded value. The decoding step maps each such encoded value pair to a floating point signal value. For 3-bit BAQ decoding there are 21 possible tables and 8 possible encoded values per table corresponding to 168 distinct mappings in the decoding table. However for 2-bit BAQ decoding there are 25 possible tables and 4 possible encoded values per table corresponding to 100 distinct mappings per decode table. The floating point decoded signal value assigned for each (table identifier, encoded value) pair is chosen to achieve unity gain assuming that samples are normally distributed with zero mean and standard deviation associated with the given table signal level.

\section{BAQ SIMULATION}

In the case of the 3-bit to 2-bit BAQ simulation, the goal is to create an alternate decode table to decode 3-bit encoded values so that the resulting decoded raw data have quantization noise with similar gain and DNR characteristics as that which would occur if the data went through standard 2-bit encoding and decoding.

This decoding table therefore must contain the same number of mapping entries as the standard 3-bit decode table but must map to different floating point values to achieve the altered noise characteristics. The approach chosen is to pairwise join adjacent BAQ encoded values and assign them the same output value. This effectively reduces the bit range of the encoding. The new output values are chosen to achieve unit gain for the samples within the merged signal ranges again assuming samples are normally distributed with zero mean and standard deviation according to the given table signal level. This concept is illustrated for a single encoding table value in Tab.1. The eight 3-bit BAQ encoded values map to eight distinct floating point values in the 3-bit decode table (denotes A-H) but only four distinct value in the 3-bit to 2-bit decode table (denoted $A^{*}$ D*).

Table 1, Mapping from 3-bit BAQ encoded value to decoding values.

\begin{tabular}{ccc}
\hline $\begin{array}{c}\text { 3-bit BAQ } \\
\text { Encoded } \\
\text { Value }\end{array}$ & $\begin{array}{c}\text { 3-bit BAQ } \\
\text { Decoding } \\
\text { Value }\end{array}$ & $\begin{array}{c}\text { 3-bit to 2-bit } \\
\text { Decoding } \\
\text { Value }\end{array}$ \\
\hline 0 & A & A* $^{*}$ \\
1 & B & A* $^{*}$ \\
2 & C & B $^{*}$ \\
3 & D & B $^{*}$ \\
4 & E & C $^{*}$ \\
5 & F & C $^{*}$ \\
6 & G & D $^{*}$ \\
7 & H & $D^{*}$ \\
\hline
\end{tabular}

\subsection{Performance evaluation of simulation method}

A Monte Carlo simulation was implemented to generate blocks of normally distributed signal values and encode/decode those using standard 2-bit tables as well as the 3-bit to 2-bit decoding method. The gain and DNR performance as a function of actual signal level are shown in Fig.2 and Fig.3. These show that the gain and DNR performance of the 3- to 2-bit downconversion are substantially similar to those demonstrated for standard 2-bit BAQ encoding/decoding. Difference in both gain and DNR are less than $0.5 \mathrm{~dB}$ over most of the signal range.

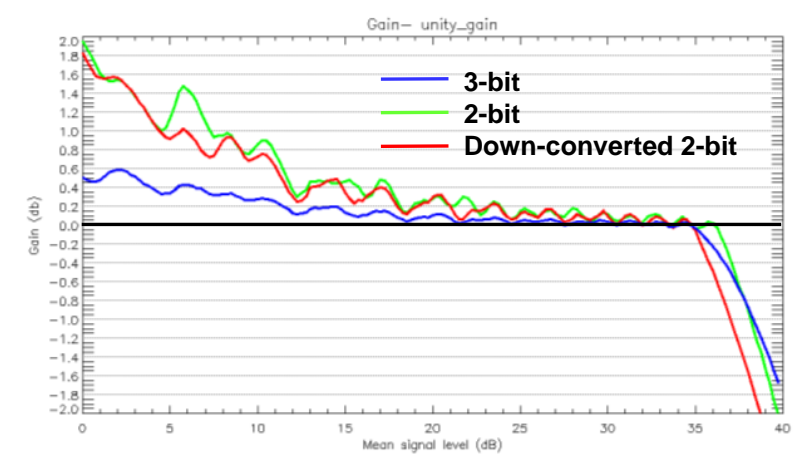

Figure 2, Gain performance comparison of standard 3bit, standard 2-bit and 3- to 2-bit down-converted BAQ. 


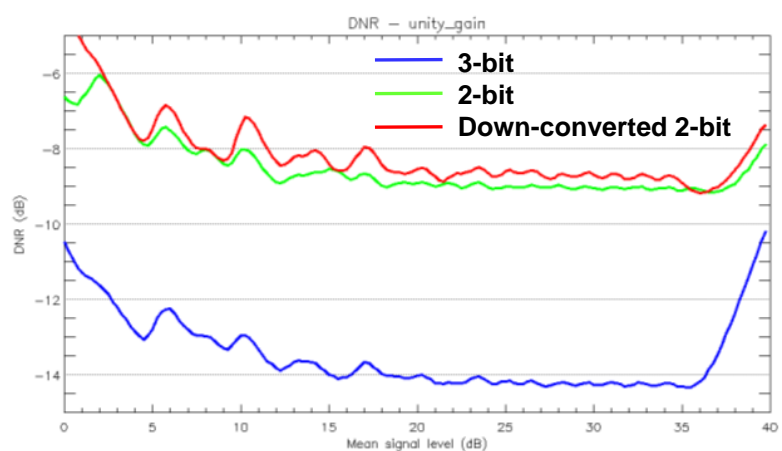

Figure 3, Digitization-noise-ratio performance comparison of standard 3-bit, standard 2-bit and 3- to 2-bit down-converted $B A Q$.

\section{STUDY CASES}

Three InSAR data stacks from distinct study areas were selected to provide some diversity of acquisition mode and terrain type: 1) arid region/high coherence case Oman (Fine mode), 2) boreal region/sparse industrial case - Alberta, Canada (Ultra-Fine mode) and 3) mixed urban case - Vancouver, Canada (Multi-Look Fine mode).

Each of these datasets consist of 20 3-bit BAQ raw format RADARSAT-2 $\mathrm{HH}$ single-polarization files processed with both standard 3-bit BAQ decode tables and the 2-bit down-conversion BAQ decode tables to derive parallel sets of SLC inputs for InSAR processing.

\section{HDS-INSAR}

To evaluate the effect of BAQ bit level on InSAR processing both the 3-bit BAQ and the 2-bit downconverted BAQ versions of all three study datasets were processed in parallel to generate multi-temporal InSAR based deformation estimates. HDS-InSAR [3] was used for processing because it is suitable for all three study cases which include natural terrain/agricultural scenes dominated by distributed scatterers and also urban/industrial scenes containing large number of phase stable persistent scatters. HDS-InSAR uses adaptive multi-looking over local neighbourhoods with statistically similar amplitude distributions [4] in order to supress noise over distributed targets while preserving point like targets. Fig.4 shows the HDSInSAR processing steps.

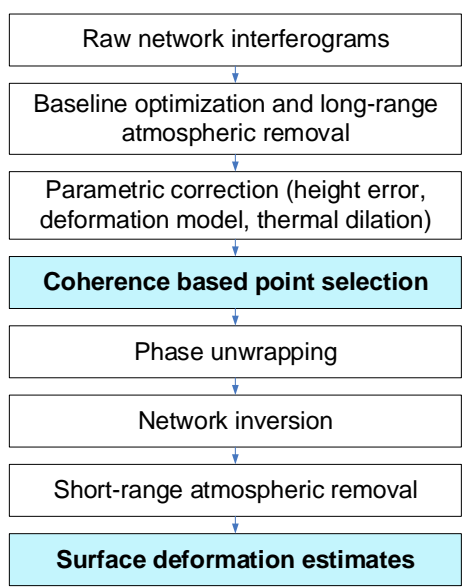

Figure 4, HDS-InSAR processing steps.

\section{RESULTS}

\subsection{Temporal Coherence}

An important step during processing is the selection of coherent targets based on the network ensemble temporal coherence as described in [5]. Spatial samples with temporal coherence exceeding a chosen quality threshold are retained to form a sparse network for further processing and subsequent deformation estimation.

Temporal coherence histograms superimposed for both 3-bit BAQ and 2-bit down-converted BAQ are shown in Fig.5. In the arid case the distributions are both skewed toward high coherence and show negligible difference between the two BAQ bit levels. In the boreal case the distributions are skewed towards low coherence and show a small shift towards lower coherence levels for 2bit BAQ when compared to 3-bit BAQ. In the mixed urban case the distributions are more uniformly distributed over the full range of coherence levels. The 2-bit BAQ distribution shows a reduction in targets with coherence > 0.4 and an increase of targets in the prominent low coherence peak near 0.15. These results are summarized in Tab.2 which lists mean temporal coherence for all targets in each case. 

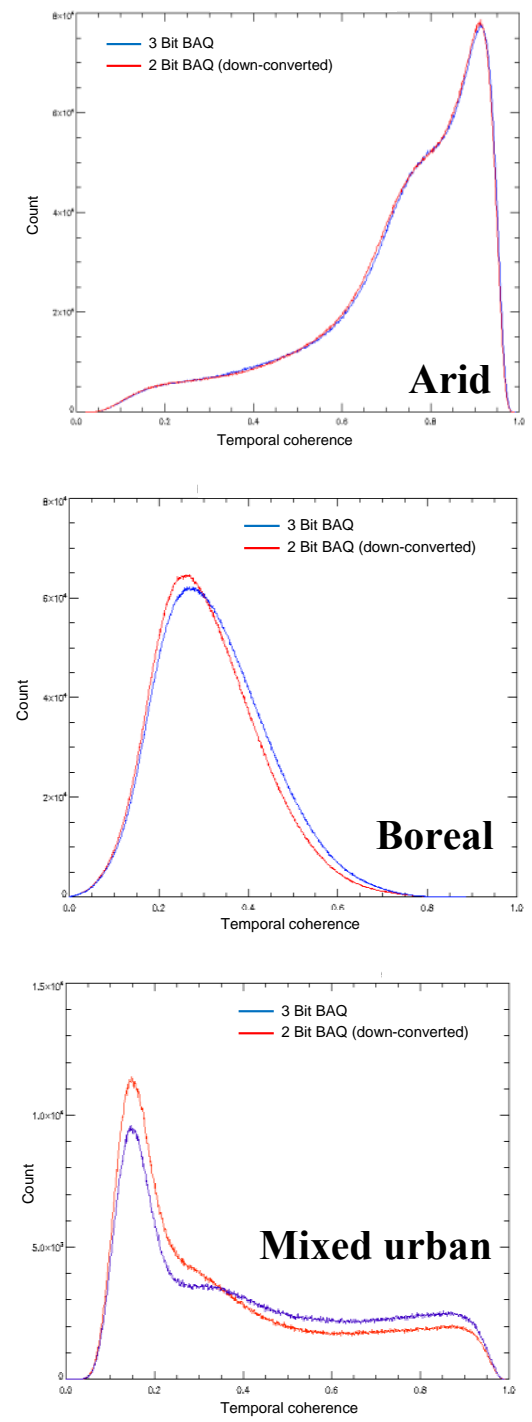

Figure 5, Comparison of 3-bit and 2-bit BAQ temporal coherence distributions.

Table 2, Summary of temporal coherence comparison.

\begin{tabular}{lccc}
\hline Case & Arid & Boreal & $\begin{array}{c}\text { Mixed } \\
\text { urban }\end{array}$ \\
\hline $\begin{array}{l}\text { Mean temporal } \\
\text { coherence (3-bit) }\end{array}$ & 0.731 & 0.298 & 0.389 \\
$\begin{array}{l}\text { Mean temporal } \\
\text { coherence (2-bit) }\end{array}$ & 0.730 & 0.285 & 0.355 \\
& & & \\
Difference & -0.001 & -0.013 & -0.034 \\
\hline
\end{tabular}

\subsection{Amplitude Analysis}

The shift of targets from high coherence to low coherence in the mixed urban case is correlated with target backscatter amplitude as shown in Fig.6. This 2D histogram shows that the distribution of per target 2-bitto-3-bit coherence ratio begins to drop significantly for low backscatter amplitudes (below $-13 \mathrm{~dB}$ ). The same ratio is rendered spatially in Fig. 7 which shows that the low coherence ratio targets correspond with the urban road network and the airport taxiways and runways. However the buildings in this case retain a near unit coherence ratio.

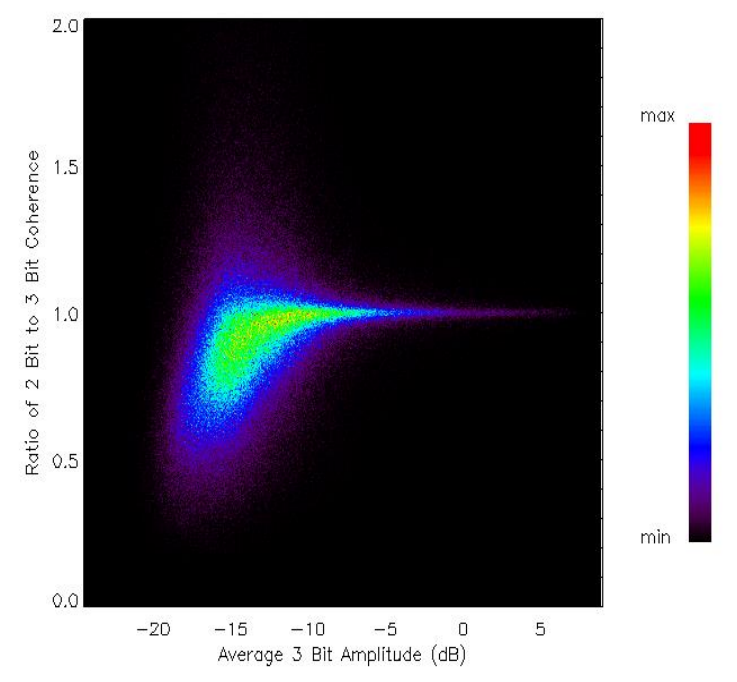

Figure 6, 2D histogram showing relationship between 2-bit to 3-bit temporal coherence ratio and target backscatter amplitude for the mixed urban case.
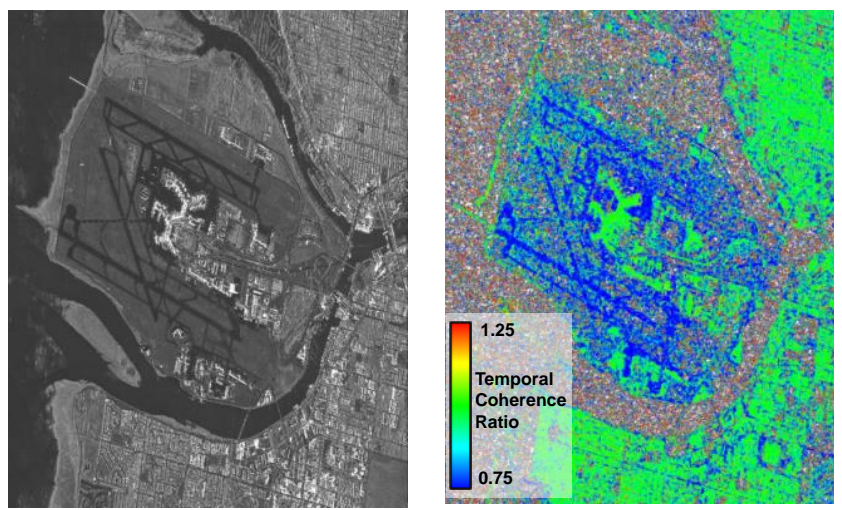

RADARSAT-2 Data and Products $\odot$ MacDonald, Dettwiler and Associates Ltd (2010). Al Rights Reserved. RADARSAT is an official mark of the Canadian Space Agency

Figure 7, Backscatter intensity image (left) and corresponding image of 2-bit to 3-bit temporal coherence ratio for the mixed urban case.

\subsection{Deformation Estimates}

Multi-temporal deformation estimates were generated for all three datasets. For each case spatial maps of the linear deformation component and a representative subset of per-target deformation time series were generated and compared. The linear deformation maps were found to be substantially similar in both the arid and boreal cases. In the mixed urban case 2-bit BAQ resulted in fewer coherent points covering asphalted 
surfaces. However the mapping of deformation over buildings and bridges is substantially similar. The time series for selected building targets agree well with differences limited to $\pm 2 \mathrm{~mm}$. Fig. 8 shows the linear deformation map and select time series for the mixed urban case centred at the Vancouver airport.
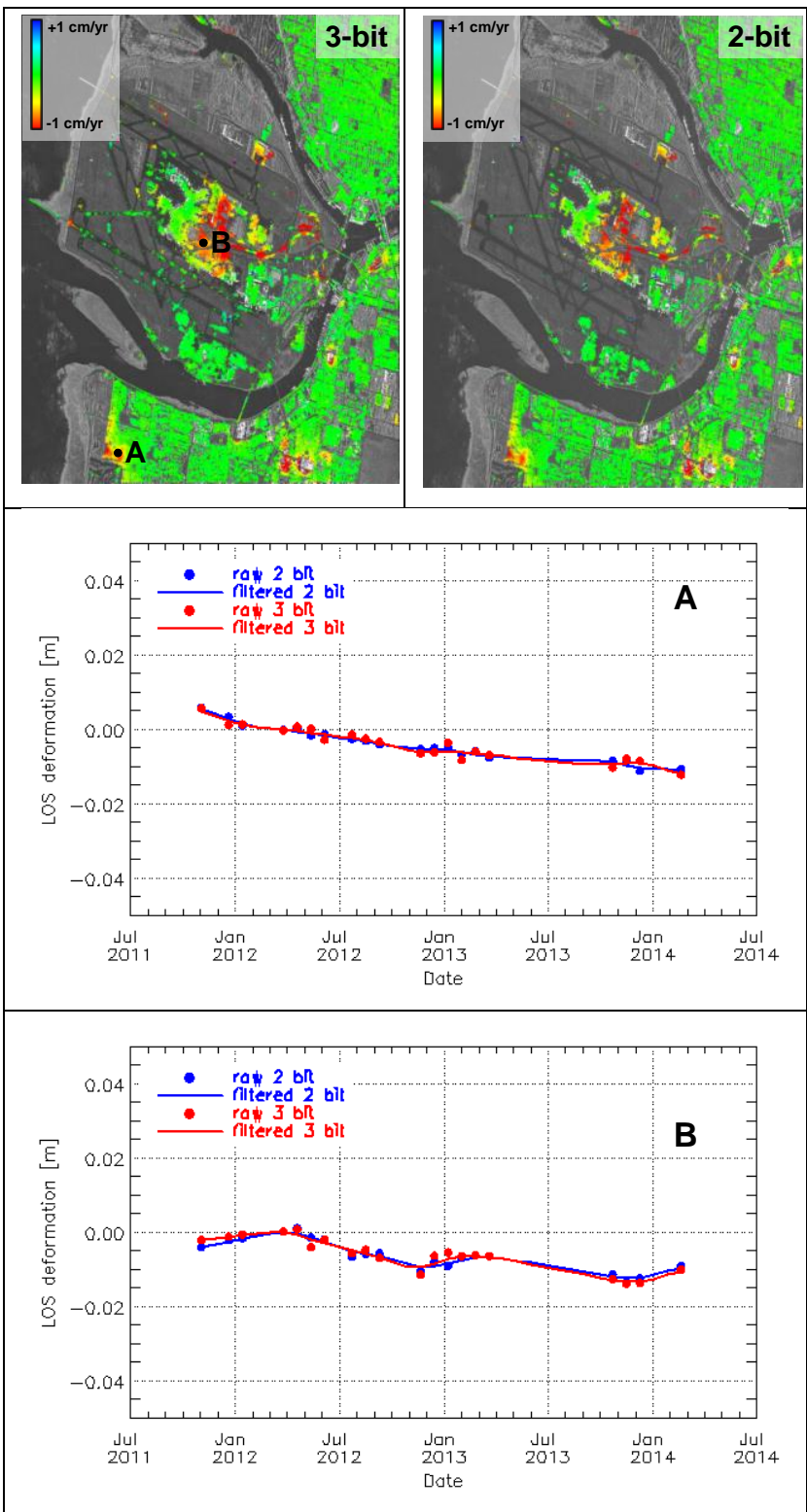

RADARSAT-2 Data and Products @ MacDonald, Dettwiler and Associates Ltd (2010). All Rights Reserved. RADARSAT is an official mark of the Canadian Space Agency

Figure 8, Comparison of 3-bit and 2-bit deformation estimates for the mixed urban case. Top row shows line-of-sight linear deformation maps for 3-bit and 2-bit $B A Q$. Lower figures show deformation time series for (A) residential house, (B) airport building.

\section{CONCLUSIONS}

This work demonstrates the relative impact of 2-bit BAQ on multi-temporal surface deformation estimation compared to 3-bit BAQ for a range of study cases. Additionally, this work shows that 2-bit BAQ SAR data can be simulated by down-conversion of 3-bit BAQ encoded data.

In the arid/high-coherence case and boreal/sparse industrial case negligible differences were found between 3-bit and corresponding 2-bit BAQ versions when comparing both temporal coherence and surface deformation estimates.

In the mixed urban case the use of 2-bit BAQ resulted in a reduction in temporal coherence for the subset of targets with low backscatter amplitude corresponding to asphalted surfaces such as roads and airport runways. This observation is consistent with expected behaviour of BAQ for low backscatter targets in a heterogeneous backscatter environment. However for other mid to high backscatter targets such as buildings and bridges the resulting deformation estimates were substantially similar for the 2-bit and 3-bit BAQ cases.

These results suggest that 2-bit BAQ data from RADARSAT-2 extended swath modes can be expected to produce substantially similar surface deformation estimates compared to those from corresponding 3-bit BAQ modes for most monitoring applications.

\section{REFERENCES}

1. RADARSAT-2 Product Description. (2014). Online at http:/gs.mdacorporation.com/includes/documents/ 52-1238_RS2_Product_Description_v1-11.pdf (as of 18 February 2015).

2. McLeod, I. \& Cumming, I. (1998). ENVISAT ASAR data reduction: Impact on SAR interferometry. IEEE Trans. Geosci. Remote Sens, 36(2), 589-602.

3. Rabus, B., Eppler, J., Sharma, J., \& Busler, J. (2012). Tunnel monitoring with an advanced InSAR technique. Proceedings of SPIE., 8361, 83611F$83611 \mathrm{~F}-10$.

4. Parizzi, A. \& Brcic, R. (2011). Adaptive InSAR Stack Multilooking Exploiting Amplitude Statistics: A Comparison Between Different Techniques and Practical Results. IEEE Geosci. Remote Sens. Letters, 8(3), 441-445.

5. Hooper, A., Zebker, H., \& Segall, P. (2004). A new method for measuring deformation on volcanoes and other natural terrains using InSAR persistent scatterers. Geophysical Research Letters, 31, L23611. 